\title{
Unravelling AR splice variant signalling in CPRC
}

Lack of response to androgen receptor (AR)-targeted therapy is a hallmark of castration-resistant prostate cancer (CRPC). A study published in Cancer Research has sought to elucidate the role of AR signalling in advanced prostate cancer, focussing on signalling mediated by AR splice variants (AR-Vs).

Androgen deprivation therapy for prostate cancer targets the full-length AR (AR-FL), which has an intact ligandbinding domain (LBD). Some therapies for CRPC, including abiraterone and MDV3100, also target AR-FL, but a renaissance in androgen signalling and clinical progression is still a problem, even with these drugs.

\section{4 ...the AR-FL and AR-Vs mediated distinct and independent transcriptional pathways... 77}

Splice variants with insertions downstream of the coding sequence for the AR DNA-binding domain, which, therefore, lack an LBD have been investigated, and it has been shown that some AR-Vs are involved in the ligand-independent AR signalling that is characteristic of CRPC. However, as AR-FL and AR-Vs are both overexpressed in CRPC, the roles of each remained unclear.

A team led by Stephen Plymate in Washington and Jun Luo at Johns Hopkins University attempted to clarify the roles of these AR forms using the prostate cancer cell lines LNCaP95 and VCP, which have similar relative AR-V expression levels to clinical specimens. Treatments targeting the LBD_including siRNA, ligand depletion and MDV3100 - suppressed AR-FL signalling, as expected, but simultaneously increased the aggregate AR-V signal and increased AR-V protein expression, suggesting a negative regulation of AR- $\mathrm{V}$ by ligand-mediated AR-FL signalling and an adaptive shift towards AR-Vmediated signalling when the AR-LBD is targeted. Furthermore, the AR-FL and AR-Vs mediated distinct and independent transcriptional pathways, with transient expression of the AR splice variant AR-V7 inducing cell cycle genes under both androgen-depleted and androgenstimulated conditions, whereas AR-FL increased expression of genes related to biosynthesis, metabolism and secretion. The absence of AR-FL did not affect the expression of genes induced by AR-V7, suggesting that AR-FL is not required for the induction of cell cycle genes by AR-Vs.

Genes in the AR-V transcriptome included UBE2C, a gene previously shown to be directly regulated by AR under androgen-deprived conditions. The data in this study suggest that UBE2C expression in CRPC is driven by AR-V but not AR-FL-in cells treated with abiraterone, UBE2C mRNA expression paralleled and was significantly associated with AR-V, but not AR-FL expression. The team further investigated these data using TURP specimens from patients who had previously had hormonal therapy, finding that AR-V7, but not AR-FL, was significantly correlated with UBE2C in at least a subset of samples.

"It is important to point out that this transcriptome is not simply the AR transcriptome generated without ligand but is a unique transcriptome consisting of genes that drive the cell through the cell cycle at a rapid rate and potentially markedly enhance tumour growth," Plymate told Nature Reviews Urology. "Indeed if you look at other tumours, the expression of UBE2C really drives tumour progression. Thus, the variant transcriptome we have described for the AR-splice variants suggests lethality and is an important target for clinical treatment."

These data provide an important insight into the AR-mediated mechanisms of castration resistance. The authors hope that the data generated will motivate the design of studies temporally analysing clinical specimens from patients before, during, and after second-line androgentargeting therapies and may eventually help to improve clinical management of patients with advanced prostate cancer.

Annette Fenner
Original article $\mathrm{Hu}$, R. et al. Distinct transcriptional programs mediated by the ligand-dependent full-length androgen receptor and its splice variants in castrationresistant prostate cancer. Cancer Res. doi:10.1158/00085472CAN-11-3892 